\title{
Attractor Bifurcation for Extended Fisher-Kolmogorov Equation
}

\author{
Honglian You, ${ }^{1}$ Rong Yuan, ${ }^{2}$ and Ziheng Zhang ${ }^{3}$ \\ ${ }^{1}$ Sino-European Institute of Aviation Engineering, Civil Aviation University of China, Tianjin 300300, China \\ ${ }^{2}$ School of Mathematical Sciences, Beijing Normal University, Beijing 100875, China \\ ${ }^{3}$ Department of Mathematics, Tianjin Polytechnic University, Tianjin 300160, China
}

Correspondence should be addressed to Honglian You; hlyou@mail.bnu.edu.cn

Received 7 July 2013; Accepted 7 August 2013

Academic Editor: Feliz Minhós

Copyright (C) 2013 Honglian You et al. This is an open access article distributed under the Creative Commons Attribution License, which permits unrestricted use, distribution, and reproduction in any medium, provided the original work is properly cited.

We consider the asymptotic stability and attractor bifurcation of the extended Fisher-Kolmogorov equation on the one-dimensional domain $(0, \pi)$ with Dirichlet or periodic boundary conditions. The novelty of this paper is that, based on a new method called attractor bifurcation, we investigate the existence of an attractor bifurcated from the trivial solution and give an explicit description of the bifurcated attractor. Moreover, the stability of the bifurcated branches is discussed.

\section{Introduction}

Fourth-order parabolic differential equations of the form

$$
\frac{\partial u}{\partial t}=-\gamma \frac{\partial^{4} u}{\partial x^{4}}+\beta \frac{\partial^{2} u}{\partial x^{2}}+f(u), \quad \gamma>0, \beta \in \mathbb{R},
$$

where $t>0, f(u)$ is a nonlinear function, arise from many physics models such as the theory of phase transitions [1], nonlinear optics [2], and shallow water waves [3]. The prototypical example of the nonlinearity $f$ is $f(u)=u-u^{3}$; that is,

$$
\frac{\partial u}{\partial t}=-\gamma \frac{\partial^{4} u}{\partial x^{4}}+\beta \frac{\partial^{2} u}{\partial x^{2}}+u-u^{3}, \quad \gamma>0, \beta \in \mathbb{R}
$$

When $\beta>0,(2)$ is usually named extended FisherKolmogorov (EFK) equation [4], while for $\beta<0$ the name Swift-Hohenberg (SH) equation [5] is more appropriate. In this paper, we restrict our attention to the case $\beta>0$. For this case, (2) is a natural extension of the classical FisherKolmogorov (FK) or Allen-Cahn equation [6, 7]. It has been proposed as a model equation for phase transitions in the neighborhood of a Lifshitz point [8] and is frequently used as a model system for the study of pattern formation from an unstable spatially homogeneous state; see $[1,4]$.

Due to the significance of fourth-order differential equations, substantial attention has been paid to (1). These results are mainly on basic properties of solutions (e.g., the existence, uniqueness, boundedness, and periodicity) [9-13], traveling waves [14], kinks [15, 16], asymptotic properties $[17,18]$, and global attractors [19]. In addition, (1) or similar equations play an important role in a variety of physics systems. Nevertheless we do not attempt to give a detailed account; instead refer the reader to [20] consulting a host of another related research.

To understand the behavior of solutions of (1), it is essential to understand the stationary (time-independent) solutions (or steady states), that is, the solutions of (1) satisfying the fourth-order differential equations of the form

$$
-\gamma u^{\prime \prime \prime \prime}+\beta u^{\prime \prime}+f(u)=0 .
$$

Typically, the stationary problem displays a multitude of periodic, homoclinic, and heteroclinic solutions. The steadystate equation of (1), that is, (3), has been analysed using shooting methods [21] and with the help of variational methods [22]. An extensive literature on this subject exists, and we refer to $[3,23-26]$ and the reference listed therein.

In the recent paper [27], based on a variational gluing method, the authors considered the set of bifurcation stationary solutions of (2) for all $L>0$ with the Neumann boundary condition; that is,

$$
\begin{aligned}
& -\gamma u^{\prime \prime \prime \prime}+\beta u^{\prime \prime}+u-u^{3}=0, \quad x \in(0, L), \\
& u_{x}(0)=u_{x x x}(0)=u_{x}(L)=u_{x x x}(L)=0 .
\end{aligned}
$$


Without loss of generality, they set $\beta=1$ and described the transition at the bifurcation point $\gamma=1 / 8$ and gave a precise description of how the attractor is changing for $\gamma=1 / 8+\epsilon$, $0<\epsilon \ll 1$ (see Section 8 in [27]).

Normally, to study (3), $\gamma, \beta$, the potential $F(F(u)=$ $\left.\int_{0}^{u} f(s) d s\right)$, the interval-length $L$, and the boundary conditions are viewed as parameters. Motivated by the above works, in this paper we introduce new parameters and study the dynamical behavior of (2). Explicitly, we focus our attention on the asymptotic behavior and attractor bifurcation (the structure and the local stability of the bifurcated steady states) of the following extended Fisher-Kolmogorov (EFK) equation with $\beta=1$ (this can be done via a variable substitution $t=\tau / \beta)$ :

$$
\frac{\partial u}{\partial t}=-\gamma \frac{\partial^{4} u}{\partial x^{4}}+\frac{\partial^{2} u}{\partial x^{2}}+\lambda u-\lambda u^{3}, \quad x \in(0, \pi),
$$

where $\gamma>0$ is a constant and $\lambda>0$ is the bifurcated parameter, using a new bifurcation theory (see Section 2) called attractor bifurcation developed recently by $\mathrm{Ma}$ and Wang $[28,29]$. We are mainly dedicated to considering (5) associated to Dirichlet boundary condition

$$
u(0, t)=u(\pi, t)=0, \quad u^{\prime \prime}(0, t)=u^{\prime \prime}(\pi, t)=0,
$$

or odd-periodic boundary condition

$$
u(x+\pi, t)=u(x, t), \quad u(-x, t)=-u(x, t),
$$

or periodic boundary condition

$$
u(x+\pi, t)=u(x, t)
$$

respectively. For the three cases, the existence and local stability of bifurcated branches are described completely (see the main results in Section 3). At the end of this paragraph, we should point out that this new bifurcation theory has been proved to be very practical. In fact, with the help of this new theory, many long-time standing bifurcation problems for a variety of PDEs arising from science and engineering, such as Boussinesq equations [30], Rayleigh-Bénard convections [31], Swift-Hohenberg (SH) equations [32], Ginzburg-Landau (GL) equations $[33,34]$, and Kuramoto-Sivashinsky equations $[28,35]$, have been extensively taken into account.

The paper is organized as follows. In Section 2, we recall some preliminaries in terms of the attractor bifurcation and the center manifold reduction process. In Section 3, the main body of this paper, we are dedicated to considering the attractor bifurcation of (5) associated to Dirichlet, odd-periodic, or periodic boundary conditions, respectively. Firstly, we calculate the corresponding eigenvalues of the linear part with different boundary conditions and determine all critical values. Then as the parameter $\lambda$ crosses the first critical value, we show that (5) bifurcates from the trivial solution an attractor $\mathscr{A}_{\lambda}$. Moreover, we demonstrate an explicit description of $\mathscr{A}_{\lambda}$ that, with Dirichlet or odd-periodic boundary conditions, it consists of two steady states, while with periodic boundary condition, it is homeomorphic to $S^{1}$. Finally, we extend these results to other critical values.

\section{Preliminary Results}

2.1. Attractor Bifurcation Theory. We first recall the general theory on attractor bifurcation of abstract evolution equations; see [28] (also [29]).

Let $H$ and $H_{1}$ be two Hilbert spaces and $H_{1} \hookrightarrow H$ a dense and compact inclusion. Consider the following abstract nonlinear evolution equation

$$
\begin{gathered}
\frac{d u}{d t}=L_{\lambda} u+G(u, \lambda), \\
u(0)=\phi,
\end{gathered}
$$

where $u:[0,+\infty) \rightarrow H$ is the unknown function, $\lambda \in \mathbb{R}$ is the system parameter, and $L_{\lambda}: H_{1} \rightarrow H$ are parameterized linear completely continuous fields continuously depending on $\lambda \in \mathbb{R}$, which satisfy

$$
\begin{aligned}
& L_{\lambda}=-A+B_{\lambda} \quad \text { a sectorial operator, } \\
& A: H_{1} \longrightarrow H \quad \text { a linear homeomorphism, } \\
& B_{\lambda}: H_{1} \longrightarrow H \quad \text { parameterized linear compact operator. }
\end{aligned}
$$

Then $L_{\lambda}$ generates an analytic semigroup $\left\{e^{-t L_{\lambda}}\right\}_{t \geq 0}$, and we can define fractional power operators $L_{\lambda}^{\alpha}$ for any $0 \leq \alpha \leq$ 1 with domain $H_{\alpha}=D\left(L_{\lambda}^{\alpha}\right)$ such that $H_{\alpha_{1}} \subset H_{\alpha_{2}}$ if $\alpha_{1}>$ $\alpha_{2}$ and $H_{0}=H$. Furthermore, we assume that the nonlinear terms $G(\cdot, \lambda): H_{\alpha} \rightarrow H$ for some $0 \leq \alpha<1$ are family of parameterized $C^{r}(r \geq 1)$ bounded operators depending continuously on the parameter $\lambda \in \mathbb{R}$ such that

$$
G(u, \lambda)=o\left(\|u\|_{H_{\alpha}}\right), \quad \forall \lambda \in \mathbb{R} .
$$

Definition 1 (see [28, Definition 5.1]). (1) We say that (9) bifurcates from $(u, \lambda)=\left(0, \lambda_{0}\right)$ an invariant set $\Omega_{\lambda}$, if there exists a sequence of invariant sets $\left\{\Omega_{\lambda_{n}}\right\}$ such that $0 \notin \Omega_{\lambda_{n}}$, and

$$
\begin{gathered}
\lim _{n \rightarrow+\infty} \lambda_{n}=\lambda_{0}, \\
\lim _{n \rightarrow+\infty} d\left(\Omega_{\lambda_{n}}, 0\right)=\lim _{n \rightarrow+\infty} \max _{u \in \Omega_{\lambda_{n}}}\|u\|_{H_{1}}=0 .
\end{gathered}
$$

(2) If the invariant sets $\Omega_{\lambda}$ are attractors of (9), then the bifurcation is called attractor bifurcation.

(3) If the invariant sets $\Omega_{\lambda}$ are attractors of (9), which are homotopic equivalent to an $m$-dimensional sphere, then we say that (9) has an $S^{m}$-attractor bifurcation at $\left(0, \lambda_{0}\right)$.

Theorem 2 (see [28, Theorem 6.1]). Assume that conditions (10) and (11) hold true. Let the eigenvalues (counting the multiplicity) of $L_{\lambda}$ be given by $\beta_{1}(\lambda), \beta_{2}(\lambda), \ldots, \beta_{k}(\lambda), \ldots \in \mathbb{C}$, where $\mathbb{C}$ is the complex space. Suppose that

$$
\begin{gathered}
\operatorname{Re} \beta_{i}(\lambda) \begin{cases}<0, & \text { if } \lambda<\lambda_{0}, \\
=0, & \text { if } \lambda=\lambda_{0}, \quad 1 \leq i \leq m, \\
>0 & \text { if } \lambda>\lambda_{0},\end{cases} \\
\operatorname{Re} \beta_{i}\left(\lambda_{0}\right)<0, \quad i \geq m+1 .
\end{gathered}
$$


Let the eigenspace of $L_{\lambda}$ at $\lambda_{0}$ be

$$
E_{0}=\bigcup_{i=1}^{m} \bigcup_{k=1}^{+\infty}\left\{u \in H_{1}:\left(L_{\lambda}-\beta_{i}\left(\lambda_{0}\right)\right)^{k} u=0\right\},
$$

and let $u=0$ be a locally asymptotically stable equilibrium point of (9) at $\lambda=\lambda_{0}$. Then the following assertions hold true.

(1) Equation (9) bifurcates from $(u, \lambda)=\left(0, \lambda_{0}\right)$ an attractor $\mathscr{A}_{\lambda}$ for $\lambda>\lambda_{0}$, with $m-1 \leq \operatorname{dim} \mathscr{A}_{\lambda} \leq m$, which is connected as $m \geq 2$.

(2) The attractor $\mathscr{A}_{\lambda}$ is a limit of a sequence of $m$ dimensional annulus $M_{k}$ with $M_{k+1} \subset M_{k}$; particularly if $\mathscr{A}_{\lambda}$ is a finite simplicial complex, then $\mathscr{A}_{\lambda}$ has the homotopy type of the $(m-1)$-dimensional sphere $S^{m-1}$.

(3) For any $u_{\lambda} \in \mathscr{A}_{\lambda}, u_{\lambda}$ can be expressed as

$$
u_{\lambda}=v_{\lambda}+o\left(\left\|v_{\lambda}\right\|_{H}\right), \quad v_{\lambda} \in E_{0} .
$$

(4) If the number of the equilibrium points of (9) in $\mathscr{A}_{\lambda}$ is finite, then one has the index formula

$$
\sum_{u_{i} \in \mathscr{A}_{\lambda}} \text { ind }\left[-\left(L_{\lambda}+G\right), u_{i}\right]= \begin{cases}2, & \text { if } m \text { is odd } \\ 0, & \text { if } m \text { is even }\end{cases}
$$

(5) If $u=0$ is globally asymptotically stable for (9) at $\lambda=$ $\lambda_{0}$, then for any bounded open set $U \subset H$ with $0 \in U$, there is an $\epsilon>0$ such that as $\lambda_{0}<\lambda<\lambda_{0}+\epsilon$, the attractor $\mathscr{A}_{\lambda}$ attracts $U \backslash \Gamma$ in $H$, where $\Gamma$ is the stable manifold of $u=0$ with codimension $m$. In particular, if (9) has a global attractor for all $\lambda$ near $\lambda_{0}$, then $\epsilon$ can be chosen independently of $U$.

From the previous theorem we see that the asymptotic stability of the equilibrium $u=0$ for $\lambda=\lambda_{0}$ is crucial. In the following, we state a theorem on the asymptotic stability of $u=0$ for $(9)_{\lambda=\lambda_{0}}$, that is, the critical state.

Theorem 3 (see [28, Theorems 3.16 and 3.17]). Let $L_{\lambda_{0}}$ : $H_{1} \rightarrow H$ be symmetric with eigenvalues $\left\{\beta_{k}\right\}_{k=1}^{\infty}$ satisfying

$$
\begin{aligned}
& \beta_{i}=0, \quad \text { if } 1 \leq i \leq m, \\
& \beta_{i}<0, \quad \text { if } i \geq m+1 .
\end{aligned}
$$

Suppose that $G\left(\cdot, \lambda_{0}\right): H_{1} \rightarrow H$ fulfills

$$
\left\langle G\left(u, \lambda_{0}\right), u\right\rangle<0, \quad \forall u \in H_{1}, u \neq 0,
$$

where $\langle\cdot, \cdot\rangle$ denotes the inner product of $H$. Then $u=0$ is globally asymptotically stable.

When the first eigenvalue is simple, that is, $m=1$, the following result describes the structure of bifurcated attractor more precisely.

Theorem 4 (see [28, Theorem 5.5 and Remark 6.1]). Let (10)-(14) hold true with $m=1$. Assume that $G(u, \lambda)$ is analytic at $u=0$ and $u=0$ is locally asymptotically stable for $(9)_{\lambda=\lambda_{0}}$. Then there exists an open set $U \subset H$ with $0 \in U$ such that if $\lambda>\lambda_{0}$, (9) bifurcates from $\left(0, \lambda_{0}\right)$ exactly two equilibrium points $u_{1}$ and $u_{2} \in U$, and the open set $U$ is decomposed into two open sets $U_{1}^{\lambda}$ and $U_{2}^{\lambda}$, satisfying the following properties:

(1) $\bar{U}=\bar{U}_{1}^{\lambda}+\bar{U}_{2}^{\lambda}, U_{1}^{\lambda} \cap U_{2}^{\lambda}=\emptyset$,

(2) $0 \in \partial U_{1}^{\lambda} \cap \partial U_{2}^{\lambda}, u_{i} \in U_{i}^{\lambda}, \quad i=1,2$,

(3) for any $\phi \in U_{i}^{\lambda}, \quad i=1,2$,

$$
\lim _{t \rightarrow+\infty}\left\|u(t, \phi)-u_{i}\right\|_{H}=0
$$

where $u(t, \phi)$ is the solution of (9) initiated with $\phi$.

If $m=1 \mathrm{in}(13)$ and condition (14) is replaced by

$$
\begin{gathered}
\operatorname{Re} \beta_{i}\left(\lambda_{0}\right)>0, \quad \text { if } 1<i \leq n+1, \\
\operatorname{Re} \beta_{i}\left(\lambda_{0}\right)<0, \quad \text { if } i>n+1,
\end{gathered}
$$

the bifurcated singular points of (9) are saddle points, of which the Morse index is obtained explicitly. In the following, we first give the definition of Morse index for nondegenerate singular points.

Definition 5 (see [28, Definition 3.5]). Let $u_{0} \in H_{1}$ be a nondegenerate singular point of

$$
L_{\lambda} u+G(u, \lambda)=0
$$

and all eigenvalues of

$$
L_{\lambda}+D_{u}\left(u_{0}, \lambda\right): H_{1} \longrightarrow H
$$

have nonzero real part. Then the Morse index of $u_{0}$ is defined by "the number of eigenvalues of (23) having positive real part."

Theorem 6 (see [28, Theorem 6.6]). Assume that (13), (21), and

$$
G(u, \lambda)=G_{k}(u, \lambda)+o\left(\|u\|^{k}\right), \quad k \geq 2 \text { an integer }
$$

hold and

$$
\alpha:=\left\langle G_{k}\left(e_{1}, \lambda_{0}\right), e_{1}\right\rangle \neq 0,
$$

where $e_{1}$ is the eigenvector of $L_{\lambda}$ corresponding to $\beta_{1}(\lambda)$. Then the bifurcated singular points of (9) from $\left(0, \lambda_{0}\right)$ have Morse index $n+1$ on $\lambda<\lambda_{0}$ and have Morse index $n$ on $\lambda>\lambda_{0}$. Moreover, $u=0$ has Morse index $n$ on $\lambda<\lambda_{0}$ and has Morse index $n+1$ on $\lambda>\lambda_{0}$.

If the multiplicity of the first eigenvalue is 2 , then there exists a 2-dimensional center manifold, and we can reduce the infinite dimensional system to this center manifold to investigate the attractor bifurcation. 
Theorem 7 (see [28, Theorem 5.10]). Let $v$ be a 2-dimensional $C^{r}(r \geq 1)$ vector field given by

$$
v_{\lambda}(y)=\lambda y-G(y, \lambda)
$$

for $y \in \mathbb{R}^{2}$. Here

$$
G(y, \lambda)=G_{k}(y, \lambda)+o\left(|y|^{k}\right),
$$

where $G_{k}(y, \lambda):=G_{k}(\underbrace{y, \ldots, y}_{k}, \lambda)$ is a $k$-multilinear field, which satisfies

$$
C_{1}|y|^{k+1} \leq\left\langle G_{k}(y, \lambda), y\right\rangle \leq C_{2}|y|^{k+1},
$$

for some constants $C_{2}>C_{1}>0, k=2 p+1$, and $p \geq 1$. Then $v_{\lambda}$ bifurcates from $(x, \lambda)=(0,0)$ on $\lambda>0$ an attractor $\mathscr{A}_{\lambda}$, which is homeomorphic to $S^{1}$. Moreover, one and only one of the following is true:

(1) $\mathscr{A}_{\lambda}$ is a periodic orbit,

(2) $\mathscr{A}_{\lambda}$ consists of only singular points, or

(3) $\mathscr{A}_{\lambda}$ contains at most $2(k+1)=4(p+1)$ singular points and has $4 N+n(N+n \geq 1)$ singular points, $2 N$ of which are saddle points, $2 \mathrm{~N}$ of which are stable node points (possibly degenerate), and $n$ of which have index zero.

2.2. Reduction Procedure. During dealing with the bifurcation problem, the reduction of the equation to its local center manifold plays a crucial role; see [28, Section 3.2] for more information. Consider (9) and assume that eigenvalues of $L_{\lambda}$ satisfy

$$
\begin{gathered}
\operatorname{Re} \beta_{i}(\lambda) \begin{cases}<0, & \text { if } \lambda<\lambda_{0}, \\
=0, & \text { if } \lambda=\lambda_{0}, \quad 1 \leq i \leq m, \\
>0, & \text { if } \lambda>\lambda_{0},\end{cases} \\
\operatorname{Re} \beta_{i}\left(\lambda_{0}\right) \neq 0, \quad i \geq m+1, \\
G(u, \lambda)=\sum_{n=k}^{\infty} G_{n}(u, \lambda),
\end{gathered}
$$

for some $k \geq 2$, where $G_{n}: H_{1} \times \cdots \times H_{1} \rightarrow H$ is an $n$ multilinear mapping and $G_{n}(u, \lambda)=G_{n}(u, \ldots, u, \lambda)$.

Let $\left\{e_{i}(\lambda)\right\}_{i=1}^{\infty}$ be the eigenvectors corresponding to $\left\{\beta_{i}(\lambda)\right\}_{i=1}^{\infty}$. Under the above assumptions there is a center manifold near $\lambda=\lambda_{0}$ with the center manifold function given by

$$
\Phi(\cdot, \lambda): \Omega \longrightarrow E_{m}^{\perp}
$$

where $\Omega \subset E_{m}$ is a neighborhood of $u=0$ and

$$
\begin{aligned}
E_{m} & =\left\{\sum_{i=1}^{m} x_{i} e_{i}(\lambda):\left(x_{1}, \ldots, x_{m}\right) \in \mathbb{R}^{m}\right\}, \\
E_{m}^{\perp} & =\left\{u \in H:\left\langle u, e_{i}(\lambda)\right\rangle=0,1 \leq i \leq m\right\} .
\end{aligned}
$$

Then (9) can be reduced to the center manifold in the following form:

$$
\frac{d x}{d t}=J_{m \lambda} x+g(x, \lambda),
$$

where $x=\left(x_{1}, \ldots, x_{m}\right)^{T} \in \mathbb{R}^{m}, J_{m \lambda}$ is the Jordan matrix corresponding to eigenvalues $\beta_{1}(\lambda), \ldots, \beta_{m}(\lambda)$ of $L_{\lambda}$, and

$$
\begin{aligned}
g(x, \lambda)=\left(\frac{1}{\left\langle e_{1}(\lambda), e_{1}(\lambda)\right\rangle}\left\langle G(x+\Phi(x, \lambda), \lambda), e_{1}(\lambda)\right\rangle\right. \\
\\
\quad \ldots, \frac{1}{\left\langle e_{m}(\lambda), e_{m}(\lambda)\right\rangle} \\
\left.\quad \times\left\langle G(x+\Phi(x, \lambda), \lambda), e_{m}(\lambda)\right\rangle\right)^{T} .
\end{aligned}
$$

By (30), we get the first approximation of (33), which is given by

$$
\frac{d x}{d t}=J_{m \lambda} x+F_{k}(x, \lambda)+o\left(|x|^{k}\right)
$$

where

$$
\begin{aligned}
F_{k}(x, \lambda) & \\
= & \left(\frac{1}{\left\langle e_{1}(\lambda), e_{1}(\lambda)\right\rangle}\left\langle G_{k}\left(\sum_{i=1}^{m} x_{i} e_{i}(\lambda), \lambda\right), e_{1}(\lambda)\right\rangle,\right. \\
& \ldots, \frac{1}{\left\langle e_{m}(\lambda), e_{m}(\lambda)\right\rangle} \\
& \left.\times\left\langle G_{k}\left(\sum_{i=1}^{m} x_{i} e_{i}(\lambda), \lambda\right), e_{m}(\lambda)\right\rangle\right)^{T} .
\end{aligned}
$$

\section{Attractor Bifurcation of the EFK Equations}

3.1. Dirichlet Boundary Condition. Consider the extended Fisher-Kolmogorov equation (5) with the Dirichlet boundary condition

$$
\begin{gathered}
\frac{\partial u}{\partial t}=-\gamma \frac{\partial^{4} u}{\partial x^{4}}+\frac{\partial^{2} u}{\partial x^{2}}+\lambda u-\lambda u^{3}, \quad x \in(0, \pi), \lambda>0, \\
u(0, t)=u(\pi, t)=0, \quad u^{\prime \prime}(0, t)=u^{\prime \prime}(\pi, t)=0, \\
u(x, 0)=u_{0}(x) .
\end{gathered}
$$

In order to apply the bifurcation theory introduced in Section 2 to discuss the attractor bifurcation of (37), firstly we choose the Hilbert spaces $H_{1}$ and $H$ as follows:

$$
\begin{gathered}
H_{1}:=\left\{u \in H^{4}(0, \pi): u(0)=u(\pi)=0,\right. \\
\left.u^{\prime \prime}(0)=u^{\prime \prime}(\pi)=0\right\}, \\
H:=L^{2}(0, \pi),
\end{gathered}
$$


and denote by $\|\cdot\|_{H}$ the norm on $H$ which is induced by the inner product

$$
\langle u, v\rangle=\int_{0}^{\pi} u(x) v(x) d x, \quad \forall u, v \in H .
$$

It is well known that the embedding of $H_{1} \hookrightarrow H$ is compact and dense. Then (37) can be transformed into the following abstract evolution equation in $H$ :

$$
\begin{gathered}
\frac{d u}{d t}=L_{\lambda} u+G(u, \lambda), \\
u(0)=u_{0},
\end{gathered}
$$

where $L_{\lambda}:=-A+B_{\lambda}$, for all $\lambda>0$, with $A$ and $B_{\lambda}: H_{1} \rightarrow H$ defined as follows:

$$
\begin{gathered}
A u=\gamma \frac{\partial^{4} u}{\partial x^{4}}-\frac{\partial^{2} u}{\partial x^{2}} \\
B_{\lambda} u=\lambda u
\end{gathered}
$$

and, for some $\alpha \in[0,1), G(\cdot, \lambda): H_{\alpha} \rightarrow H$ is of the form

$$
G(u, \lambda)=-\lambda u^{3} \text {. }
$$

An easy calculation shows that the eigenvalues of $-A: H_{1} \rightarrow$ $H$ are

$$
\delta_{n}=-n^{2}\left(1+n^{2} \gamma\right), \quad n=1,2, \ldots
$$

with eigenvectors $e_{n}(x)=\sin n x$. Hence, $-A$ is a linear homeomorphism. On the other hand, since $H_{1}$ is compactly imbedded in $H, B_{\lambda}$ is a compact operator. Therefore, $L_{\lambda}=$ $-A+B_{\lambda}$ is a linear completely continuous filed.

Proposition 8. For any $\lambda>0, L_{\lambda}$ is a sectorial operator.

Proof. It is obvious that the spectrum of $-A$ consists of eigenvalues; that is,

$$
\sigma(-A)=\left\{-n^{2}\left(1+n^{2} \gamma\right), n=1,2, \ldots\right\} .
$$

Let $u=\sum_{n=1}^{\infty} u_{n} e_{n}$. Then

$$
(\delta I+A)^{-1} u=\sum_{n=1}^{\infty}\left(\delta-\delta_{n}\right)^{-1} u_{n} e_{n}, \quad \text { if } \delta>\delta_{1},
$$

which yields that

$$
\left\|(\delta I+A)^{-1}\right\| \leq \frac{1}{\delta-\delta_{1}}, \quad \text { if } \delta>\delta_{1} .
$$

Therefore, by [36, page 12, Corollary 3.8],

$$
\|T(t)\| \leq e^{\delta_{1} t} \leq 1, \quad \forall t \geq 0,
$$

where $T(t), t \geq 0$, is the $C_{0}$-semigroup generated by $-A$. On the other hand, for $\delta \in \mathbb{C}$ with $\operatorname{Re} \delta>0$ and $\operatorname{Im} \delta \neq 0$, we have

$$
\left\|(\delta I+A)^{-1}\right\| \leq \frac{1}{\left|\delta-\delta_{1}\right|} \leq \frac{1}{|\operatorname{Im} \delta|} .
$$

By [36, page 61, Theorem 5.2], $-A$ is a sectorial operator. Furthermore, $B_{\lambda}: H_{1} \rightarrow H$ is a linear bounded operator. Therefore, $-A+B_{\lambda}$ is a sectorial operator.
Lemma 9. For any $1 / 8<\alpha<1, G(u, \lambda)=o\left(\|u\|_{H_{\alpha}}\right)$, for all $\lambda>0$.

Proof. For any $u \in H_{\alpha}$, by [37, Theorem 1.6.1], we have

$$
\|G(u, \lambda)\|_{H}^{2}=\lambda^{2} \int_{0}^{\pi} u^{6} d x \leq \pi \lambda^{2}\|u\|_{C^{0}}^{6} \leq C_{1} 2 \pi \lambda^{2}\|u\|_{H_{\alpha}}^{6},
$$

where $\|\cdot\|_{C^{0}}$ denotes the supremum norm on $[0, \pi], C_{1}>0$ is some constant, and $\alpha \in[0,1)$ satisfies $4 \alpha-1 / 2>0$, that is, $1 / 8<\alpha<1$.

From the hypotheses in Theorem 2, we know that the eigenvalues of $L_{\lambda}$ are vital to give rise to the bifurcation. After a simple computation, the eigenvalues and the eigenvectors of $L_{\lambda}$ are given as follows.

Proposition 10. The eigenvalues of $L_{\lambda}$ are

$$
\beta_{n}(\lambda)=\lambda-n^{2}\left(1+n^{2} \gamma\right), \quad n=1,2, \ldots,
$$

and the corresponding eigenvectors are

$$
e_{n}(x)=\sin n x, \quad n=1,2, \ldots
$$

Moreover, $\left\{e_{n}(x)\right\}_{n=1}^{\infty}$ forms an orthogonal basis of $H_{1}$, as well as $H$.

From (50) we know that every eigenvalue is simple and the first eigenvalue is $\beta_{1}(\lambda)=\lambda-(1+\gamma)$. Take the first critical value $\lambda_{1}:=1+\gamma$. Now we give the result on bifurcation for (37) when $\lambda$ crosses $\lambda_{1}$.

Theorem 11. For (37), one has the following assertions.

(1) As $0<\lambda \leq 1+\gamma, u=0$ is globally asymptotically stable.

(2) Equation (37) bifurcates from $(0,1+\gamma)$ an attractor $\mathscr{A}_{\lambda}$ on the right side of $\lambda=1+\gamma$, which consists of exactly two steady states $u_{1}^{\lambda}$ and $u_{2}^{\lambda}$ expressed as follows:

$$
\begin{gathered}
u_{1}^{\lambda}=\alpha(\lambda) \sin x+o(\alpha(\lambda)), \\
u_{2}^{\lambda}=-\alpha(\lambda) \sin x+o(\alpha(\lambda)), \\
\alpha(\lambda)=\sqrt{\frac{4(\lambda-(1+\gamma))}{3 \lambda} .}
\end{gathered}
$$

(3) For some $\epsilon>0$, if $1+\gamma<\lambda<1+\gamma+\epsilon$, there exists an open set $U \subset H$ containing 0 , which can be decomposed by the stable manifold of $u=0$ into two open sets $U_{1}^{\lambda}$ and $U_{2}^{\lambda}$ with the property that $u_{i}^{\lambda} \in U_{i}^{\lambda}, i=1,2$, and

$$
\lim _{t \rightarrow+\infty}\left\|u(t, \phi)-u_{i}^{\lambda}\right\|_{H}=0, \quad \phi \in U_{i}^{\lambda},
$$

where $u(t, \phi)$ is the solution of (37) initiated with $\phi$.

Proof. For any $0 \neq u \in H_{1}$ and $\lambda>0$,

$$
\langle G(u, \lambda), u\rangle=-\lambda \int_{0}^{\pi} u^{4} d x<0 .
$$


By Theorem 3, $u=0$ is globally asymptotically stable as $0<$ $\lambda \leq 1+\gamma$.

The existence of $\mathscr{A}_{\lambda}$ follows from Propositions 8 and 10, Lemma 9, and Theorem 2 directly. Moreover, Theorem 4 indicates that the bifurcated attractor consists of exactly two steady states and (3) is also true. Now we give the formula of two steady states by reducing (40) to its local center manifold.

Let $u(x)=\sum_{n=1}^{\infty} u_{n} \sin n x \in H_{1}$. Then

$$
\frac{d u_{n}}{d t}=\beta_{n}(\lambda) u_{n}+\frac{2}{\pi}\langle G(u, \lambda), \sin n x\rangle,
$$

which deduces that the steady-state equation is of the form

$$
\beta_{n}(\lambda) u_{n}+\frac{2}{\pi}\langle G(u, \lambda), \sin n x\rangle=0
$$

Since

$$
G(u, \lambda)=-\lambda u^{3}=: G_{3}(u, \lambda),
$$

where $G_{3}: H_{1} \times H_{1} \times H_{1} \rightarrow H$ is a 3-multilinear mapping, we have

$$
\begin{aligned}
& \langle G(u, \lambda), \sin n x\rangle \\
& =\left\langle G_{3}\left(\sum_{i=1}^{\infty} u_{i} \sin i x, \sum_{j=1}^{\infty} u_{j} \sin j x, \sum_{k=1}^{\infty} u_{k} \sin k x\right), \sin n x\right\rangle \\
& =\sum_{i, j, k=1}^{\infty} u_{i} u_{j} u_{k}\left\langle G_{3}(\sin i x, \sin j x, \sin k x, \lambda), \sin n x\right\rangle \\
& =-\lambda \sum_{i, j, k=1}^{\infty} u_{i} u_{j} u_{k} \int_{0}^{\pi}(\sin i x \sin j x \sin k x) \sin n x d x \\
& =\frac{\lambda}{4} \sum_{i, j, k=1}^{\infty} u_{i} u_{j} u_{k} \int_{0}^{\pi}(\sin (i+j+k) x-\sin (i+j-k) x \\
& -\sin (i-j+k) x \\
& -\sin (j+k-i) x) \sin n x d x
\end{aligned}
$$

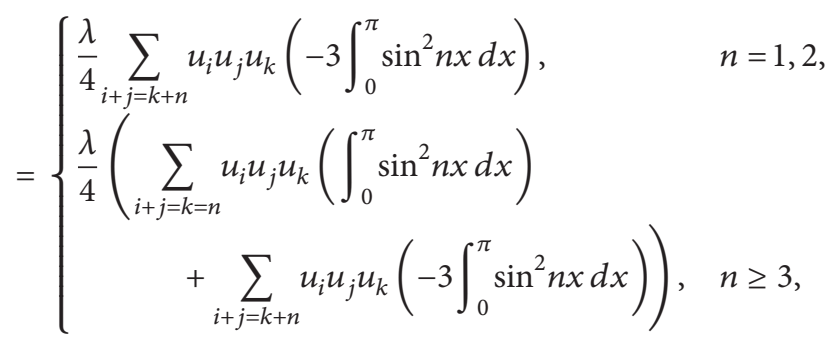

$$
\begin{aligned}
& = \begin{cases}-\frac{3 \pi \lambda}{8} \sum_{i+j=k+n} u_{i} u_{j} u_{k}, & n=1,2, \\
-\frac{\pi \lambda}{8}\left(3 \sum_{i+j=k+n} u_{i} u_{j} u_{k}-\sum_{i+j=k=n} u_{i} u_{j} u_{k}\right), & n \geq 3 .\end{cases}
\end{aligned}
$$

For $n=1$ in (56), we have

$$
\beta_{1}(\lambda) u_{1}-\frac{2}{\pi} \cdot \frac{3 \pi \lambda}{8} \sum_{i+j=k+1} u_{i} u_{j} u_{k}=0
$$

which yields that

$$
u_{1}=\frac{3 \lambda}{4 \beta_{1}(\lambda)} \sum_{i+j=k+1} u_{i} u_{j} u_{k}
$$

Similarly, one deduces that

$$
\begin{gathered}
u_{2}=\frac{3 \lambda}{4 \beta_{2}(\lambda)} \sum_{i+j=k+2} u_{i} u_{j} u_{k}, \\
u_{3}=\frac{\lambda}{4 \beta_{3}(\lambda)}\left(3 \sum_{i+j=k+3} u_{i} u_{j} u_{k}-u_{1}^{3}\right), \\
u_{n}=\frac{\lambda}{4 \beta_{n}(\lambda)}\left(3 \sum_{i+j=k+n} u_{i} u_{j} u_{k}-\sum_{i+j+k=n} u_{i} u_{j} u_{k}\right), n \geq 4 .
\end{gathered}
$$

Hence, by induction, we have

$$
\begin{gathered}
u_{2}=o\left(\left|u_{1}\right|^{3}\right), \\
u_{3}=-\frac{\lambda}{4 \beta_{3}(\lambda)} u_{1}^{3}+o\left(\left|u_{1}\right|^{3}\right), \\
u_{n}=c_{n} u_{1}^{n}+o\left(\left|u_{1}\right|^{n}\right), \quad n \geq 4,
\end{gathered}
$$

where $c_{n}$ is a constant. Then we obtain the steady-state bifurcation equation of (37) as follows:

$$
4 \beta_{1}(\lambda) u_{1}-3 \lambda u_{1}^{3}+o\left(\left|u_{1}\right|^{3}\right)=0,
$$

and after an easy computation, we have

$$
u_{1}= \pm \sqrt{\frac{4(\lambda-(1+\gamma))}{3 \lambda}}+o\left(\left|u_{1}\right|\right)
$$

By (3) in Theorem 2, the elements in the bifurcated attractor $\mathscr{A}_{\lambda}$ are

$$
\begin{aligned}
u_{1} \sin x+o\left(\left\|u_{1} \sin x\right\|_{H}\right)= & \pm \sqrt{\frac{4(\lambda-(1+\gamma))}{3 \lambda} \sin x} \\
& +o\left(\sqrt{\frac{4(\lambda-(1+\gamma))}{3 \lambda}}\right) .
\end{aligned}
$$

As $\lambda$ crosses the $n$th critical value $\lambda_{n}=n^{2}\left(1+n^{2} \gamma\right)$, $n=2,3, \ldots$, the local stability of the bifurcated steady states does not hold true. Nevertheless, as a direct consequence of Theorem 6, we have the following result.

Theorem 12. Equation (37) bifurcates from $\left(0, n^{2}\left(1+n^{2} \gamma\right)\right)$, $n=2,3, \ldots$, two saddle points with Morse index $n$ on $\lambda<$ $n^{2}\left(1+n^{2} \gamma\right)$ and Morse index $n-1$ on $\lambda>n^{2}\left(1+n^{2} \gamma\right)$. 
3.2. Periodic Boundary Condition. In this section we are concerned with two cases: the odd-periodic boundary condition or the periodic boundary condition. Now we deal with the first case

$$
\begin{gathered}
\frac{\partial u}{\partial t}=-\gamma \frac{\partial^{4} u}{\partial x^{4}}+\frac{\partial^{2} u}{\partial x^{2}}+\lambda u-\lambda u^{3}, \quad x \in(0, \pi), \lambda>0 \\
u(x, t)=u(x+\pi, t), \quad u(-x, t)=-u(x, t), \\
u(x, 0)=u_{0}(x) .
\end{gathered}
$$

Here we choose the Hilbert spaces $\widetilde{H}_{1}$ and $\widetilde{H}$ as follows:

$$
\begin{gathered}
\widetilde{H}_{1}=\left\{u \in H^{4}(0, \pi): u(x)=u(x+\pi),\right. \\
\left.u(-x)=-u(x), \int_{0}^{\pi} u(x) d x=0\right\}, \\
\widetilde{H}=\left\{u \in L^{2}(0, \pi): u(x)=u(x+\pi),\right. \\
\left.u(-x)=-u(x), \int_{0}^{\pi} u(x) d x=0\right\} .
\end{gathered}
$$

It is obvious that $\widetilde{H}_{1} \hookrightarrow \widetilde{H}$ is compact and dense. Similar to that in Section 3.1, $A$ and $B_{\lambda}$ are defined by

$$
\begin{gathered}
A u=\gamma \frac{\partial^{4} u}{\partial x^{4}}-\frac{\partial^{2} u}{\partial x^{2}} \\
B_{\lambda} u=\lambda u
\end{gathered}
$$

and, for some $\alpha \in[0,1), G(\cdot, \lambda): \widetilde{H}_{\alpha} \rightarrow \widetilde{H}$ is of the form

$$
G(u, \lambda)=-\lambda u^{3}
$$

Denote $L_{\lambda}:=-A+B_{\lambda}$, for all $\lambda>0$. Then (66) can be deformed into the following abstract evolution equation in $\widetilde{H}_{1}$ :

$$
\begin{gathered}
\frac{d u}{d t}=L_{\lambda} u+G(u, \lambda), \\
u(0)=u_{0},
\end{gathered}
$$

in which $L_{\lambda}$ is a linear completely continuous field and a sectorial operator and symmetric and $G(u, \lambda)=o\left(\|u\|_{\widetilde{H}_{\alpha}}\right)(1 / 8<$ $\alpha<1$ ), for all $\lambda>0$.

Proposition 13. The eigenvalues of $L_{\lambda}$ in $\widetilde{H}$ are

$$
\beta_{n}(\lambda)=\lambda-4 n^{2}\left(1+4 \gamma n^{2}\right), \quad n=1,2, \ldots,
$$

and the corresponding eigenvectors are

$$
e_{n}(x)=\sin 2 n x, \quad n=1,2, \ldots
$$

In addition, $\left\{e_{n}(x)\right\}_{n=1}^{\infty}$ forms an orthogonal basis of $\widetilde{H}_{1}$, as well as $\widetilde{H}$.

Now we are in the position to state the bifurcation result for (66) at the first eigenvalue.

Theorem 14. For (66), the following assertions hold true.

(1) For $0<\lambda \leq 4(1+4 \gamma), u=0$ is globally asymptotically stable.

(2) Equation (66) bifurcates from $(0,4(1+4 \gamma))$, on the right side of $\lambda=4(1+4 \gamma)$, an attractor $\mathscr{A}_{\lambda}$, which consists of exactly two steady states $u_{1}^{\lambda}$ and $u_{2}^{\lambda}$, expressed by

$$
\begin{aligned}
& u_{1}^{\lambda}=\alpha(\lambda) \sin 2 x+o(\alpha(\lambda)), \\
& u_{2}^{\lambda}=-\alpha(\lambda) \sin 2 x+o(\alpha(\lambda)), \\
& \alpha(\lambda)=\sqrt{\frac{4(\lambda-4(1+4 \gamma))}{3 \lambda} .}
\end{aligned}
$$

(3) For some $\epsilon>0$, if $4(1+4 \gamma)<\lambda<4(1+4 \gamma)+\epsilon$, there exists an open set $U \subset H$ containing 0 , which can be decomposed by the stable manifold of $u=0$ into two open sets $U_{1}^{\lambda}$ and $U_{2}^{\lambda}$ with the property that $u_{i}^{\lambda} \in U_{i}^{\lambda}$, $i=1,2$, and

$$
\lim _{t \rightarrow+\infty}\left\|u(t, \phi)-u_{i}^{\lambda}\right\|_{H}=0, \quad \phi \in U_{i}^{\lambda}
$$

where $u(t, \phi)$ is the solution of (66) initiated with $\phi$.

Proof. For the proof of (1) and the existence of $\mathscr{A}_{\lambda}$, it is just the same as in Theorem 11. In what follows, we are devoted to describing the structure of $\mathscr{A}_{\lambda}$.

Let $u \in \widetilde{H}_{1}$ be expressed as $u(x)=\sum_{n=1}^{\infty} u_{n} \sin 2 n x$. Then we obtain that

$$
\frac{d u_{n}}{d t}=\beta_{n}(\lambda) u_{n}+\frac{2}{\pi}\langle G(u, \lambda), \sin 2 n x\rangle
$$

Proposition 13 shows that the eigenvalues here are simple. Therefore, with the help of Theorem 4, we know that the bifurcated attractor consists of exactly two steady states. Hence, we consider the following steady-state equation:

$$
\beta_{n}(\lambda) u_{n}+\frac{2}{\pi}\langle G(u, \lambda), \sin 2 n x\rangle=0
$$


By (57), we get

$$
\begin{aligned}
& \langle G(u, \lambda), \sin 2 n x\rangle \\
& =\left\langle G_{3}\left(\sum_{i=1}^{\infty} u_{i} \sin 2 i x, \sum_{j=1}^{\infty} u_{j} \sin 2 j x, \sum_{k=1}^{\infty} u_{k} \sin 2 k x, \lambda\right), \sin 2 n x\right\rangle \\
& =\sum_{i, j, k=1}^{\infty} u_{i} u_{j} u_{k}\left\langle G_{3}(\sin 2 i x, \sin 2 j x, \sin 2 k x, \lambda), \sin 2 n x\right\rangle \\
& =-\lambda \sum_{i, j, k=1}^{\infty} u_{i} u_{j} u_{k} \int_{0}^{\pi}(\sin 2 i x \sin 2 j x \sin 2 k x) \sin 2 n x d x \\
& =\frac{\lambda}{4} \sum_{i, j, k=1}^{\infty} u_{i} u_{j} u_{k} \int_{0}^{\pi}(\sin 2(i+j+k) x-\sin 2(i+j-k) x \\
& -\sin 2(i-j+k) x \\
& -\sin 2(j+k-i) x) \sin 2 n x d x
\end{aligned}
$$

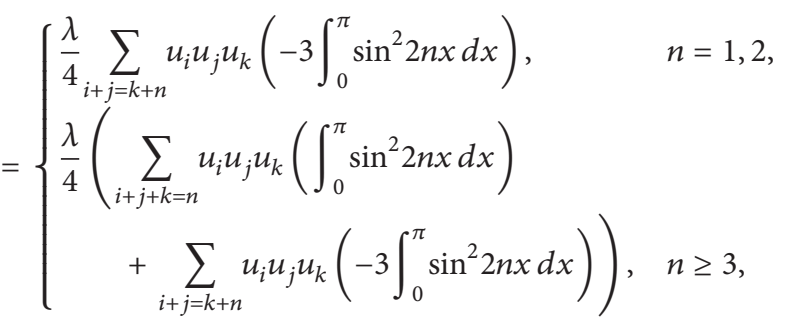

$$
\begin{aligned}
& = \begin{cases}-\frac{3 \pi \lambda}{8} \sum_{i+j=k+n} u_{i} u_{j} u_{k}, & n=1,2, \\
-\frac{\pi \lambda}{8}\left(3 \sum_{i+j=k+n} u_{i} u_{j} u_{k}-\sum_{i+j+k=n} u_{i} u_{j} u_{k}\right), & n \geq 3 .\end{cases}
\end{aligned}
$$

Similar to Theorem 11, for $n=1$, we get the bifurcation equation

$$
4 \beta_{1}(\lambda) u_{1}-3 \lambda u_{1}^{3}+o\left(\left|u_{1}\right|^{3}\right)=0
$$

The remaining part is the same as in Theorem 11. So we omit the details.

As far as the $n$th critical value is concerned, we have the following result.

Theorem 15. Equation (66) bifurcates from $\left(0,4 n^{2}\left(1+4 n^{2} \gamma\right)\right)$, $n=2,3, \ldots$, two saddle points with Morse index $n$ on $\lambda<$ $4 n^{2}\left(1+4 n^{2} \gamma\right)$ and Morse index $n-1$ on $\lambda>4 n^{2}\left(1+4 n^{2} \gamma\right)$.

In what follows, we turn our attention to the periodic boundary condition

$$
\begin{gathered}
\frac{\partial u}{\partial t}=-\gamma \frac{\partial^{4} u}{\partial x^{4}}+\frac{\partial^{2} u}{\partial x^{2}}+\lambda u-\lambda u^{3}, \quad x \in(0, \pi), \lambda>0 \\
u(x, t)=u(x+\pi, t) \\
u(x, 0)=u_{0}(x)
\end{gathered}
$$

Let

$$
\begin{aligned}
& \widehat{H}_{1}=\left\{u \in H^{4}(0, \pi): u(x)=x(x+\pi), \int_{0}^{\pi} u(x) d x=0\right\}, \\
& \widehat{H}=\left\{u \in L^{2}(0, \pi): u(x)=x(x+\pi), \int_{0}^{\pi} u(x) d x=0\right\} .
\end{aligned}
$$

Define $A$ and $B_{\lambda}$ as follows:

$$
\begin{gathered}
A u=\gamma \frac{\partial^{4} u}{\partial x^{4}}-\frac{\partial^{2} u}{\partial x^{2}} \\
B_{\lambda} u=\lambda u
\end{gathered}
$$

and, for $\alpha \in[0,1), G(\cdot, \lambda): \widehat{H}_{1} \rightarrow \widehat{H}$ is defined by

$$
G(u, \lambda)=-\lambda u^{3}
$$

Denote $L_{\lambda}:=-A+B_{\lambda}$, for all $\lambda \in \mathbb{R}$. Then (79) can be transformed into the following abstract evolution equation in $\widehat{H}_{1}$ :

$$
\begin{gathered}
\frac{d u}{d t}=L_{\lambda} u+G(u, \lambda), \\
u(0)=u_{0},
\end{gathered}
$$

in which $L_{\lambda}$ is a linear completely continuous field and a sectorial operator and symmetric, and $G(u, \lambda)=o\left(\|u\|_{\widehat{H}_{\alpha}}\right)$ $(1 / 8<\alpha<1)$, for all $\lambda>0$.

Proposition 16. The eigenvalues of $L_{\lambda}$ in $\widehat{H}$ are

$$
\beta_{2 n-1}(\lambda)=\beta_{2 n}(\lambda)=\lambda-4 n^{2}\left(1+4 \gamma n^{2}\right), \quad n=1,2, \ldots,
$$

and the corresponding eigenvectors are

$$
e_{2 n-1}(x)=\sin 2 n x, \quad e_{2 n}(x)=\cos 2 n x, \quad n=1,2, \ldots
$$

Moreover, $\left\{e_{2 n-1}(x), e_{2 n}(x)\right\}_{n=1}^{\infty}$ forms an orthogonal basis of $\widehat{H}_{1}$ and $\widehat{H}$.

From (84) we know that every eigenvalue has multiplicity 2. Consider the first critical value $\lambda_{1}=4(1+4 \gamma)>0$. Then (79) has a 2-dimensional center manifold when $\lambda=\lambda_{1}$. Reducing (79) to its center manifold and using Theorem 7, we get the following bifurcation result of $(79)$ at $\left(0, \lambda_{1}\right)$.

Theorem 17. For (79), one has the following conclusions.

(1) For $0<\lambda \leq 4(1+4 \gamma), u=0$ is globally asymptotically stable.

(2) Equation (79) bifurcates from $(0,4(1+4 \gamma))$, on the right side of $\lambda=4(1+4 \gamma)$, an attractor $\mathscr{A}_{\lambda}$. 
(3) $\mathscr{A}_{\lambda} \cong S^{1}$ consists of steady states, which can be expressed as follows:

$$
\begin{gathered}
\mathscr{A}_{\lambda}=\left\{\alpha_{1}(\lambda) \sin 2 x+\alpha_{2}(\lambda) \cos 2 x+o\left(\left|\alpha_{1}(\lambda)\right|,\left|\alpha_{2}(\lambda)\right|\right):\right. \\
\left.\alpha_{1}^{2}(\lambda)+\alpha_{2}^{2}(\lambda)=\frac{4(\lambda-4(1+4 \gamma))}{3 \lambda}>0\right\} .
\end{gathered}
$$

Proof. For the proof of (1) and (2), we can see Theorems 11 or 14 . Next we focus on proving (3). Since $u(x)=$ $\sum_{n=1}^{\infty}\left(u_{n} \sin 2 n x+v_{n} \cos 2 n x\right) \in \widehat{H}_{1}$, reducing (79) to the 2dimensional center manifold, we obtain that

$$
\begin{aligned}
& \frac{d u_{1}}{d t}=\beta_{1}(\lambda) u_{1}+\frac{2}{\pi}\langle G(u, \lambda), \sin 2 x\rangle, \\
& \frac{d v_{1}}{d t}=\beta_{1}(\lambda) v_{1}+\frac{2}{\pi}\langle G(u, \lambda), \cos 2 x\rangle .
\end{aligned}
$$

By (35), we get the first approximation of (87)

$$
\begin{aligned}
\frac{d u_{1}}{d t}= & \beta_{1}(\lambda) u_{1}+\frac{2}{\pi}\left\langle G_{3}\left(u_{1} \sin 2 x+v_{1} \cos 2 x, \lambda\right), \sin 2 x\right\rangle \\
& +o\left(|y|^{3}\right), \\
\frac{d v_{1}}{d t}= & \beta_{1}(\lambda) v_{1}+\frac{2}{\pi}\left\langle G_{3}\left(u_{1} \sin 2 x+v_{1} \cos 2 x, \lambda\right), \cos 2 x\right\rangle \\
& +o\left(|y|^{3}\right),
\end{aligned}
$$

where $y=\left(u_{1}, v_{1}\right)^{T} \in \mathbb{R}^{2}$. On the other hand,

$$
\begin{aligned}
& \left\langle G_{3}\left(u_{1} \sin 2 x+v_{1} \cos 2 x, \lambda\right), \sin 2 x\right\rangle \\
& =-\lambda \int_{0}^{\pi}\left(u_{1}^{3} \sin ^{3} 2 x+3 u_{1}^{2} v_{1} \sin ^{2} 2 x \cos 2 x\right. \\
& \left.\quad+3 u_{1} v_{1}^{2} \sin 2 x \cos ^{2} 2 x+v_{1}^{3} \cos ^{3} 2 x\right) \sin 2 x d x \\
& =-\frac{3 \pi \lambda}{8}\left(u_{1}^{3}+u_{1} v_{1}^{2}\right), \\
& \left\langle G_{3}\left(u_{1} \sin 2 x+v_{1} \cos 2 x, \lambda\right), \cos 2 x\right\rangle \\
& =-\lambda \int_{0}^{\pi}\left(u_{1}^{3} \sin ^{3} 2 x+3 u_{1}^{2} v_{1} \sin ^{2} 2 x \cos 2 x\right. \\
& \left.\quad+3 u_{1} v_{1}^{2} \sin 2 x \cos ^{2} 2 x+v_{1}^{3} \cos ^{3} 2 x\right) \cos 2 x d x \\
& =-\frac{3 \pi \lambda}{8}\left(v_{1}^{3}+v_{1} u_{1}^{2}\right) .
\end{aligned}
$$

Thus, we deduce that

$$
\begin{aligned}
& \frac{d u_{1}}{d t}=\beta_{1}(\lambda) u_{1}-\frac{3 \lambda}{4}\left(u_{1}^{3}+u_{1} v_{1}^{2}\right)+o\left(|y|^{3}\right), \\
& \frac{d v_{1}}{d t}=\beta_{1}(\lambda) v_{1}-\frac{3 \lambda}{4}\left(v_{1}^{3}+v_{1} u_{1}^{2}\right)+o\left(|y|^{3}\right),
\end{aligned}
$$

where $y=\left(u_{1}, v_{1}\right)^{T} \in \mathbb{R}^{2}$. Let

$$
\widetilde{G}(y, \lambda)=\frac{3 \lambda}{4}\left(\begin{array}{c}
u_{1}^{3}+u_{1} v_{1}^{2} \\
v_{1}^{3}+v_{1} u_{1}^{2}
\end{array}\right)+o\left(|y|^{3}\right) .
$$

Then $\widetilde{G}(y, \lambda)$ satisfies Theorem 7 with $k=3$ and $0<C_{1}<$ $3 \lambda / 4<C_{2}$. Thus, if $\lambda>4(1+4 \gamma)$, (79) bifurcates an attractor $\mathscr{A}_{\lambda} \cong S^{1}$.

Finally, we prove that the attractor $\mathscr{A}_{\lambda}$ consists of steady states. It is clear that $\widetilde{H}_{1} \subset \widehat{H}_{1}, \widetilde{H} \subset \widehat{H}$, and $\left(L_{\lambda}+\right.$ $G(\cdot, \lambda)) \widetilde{H}_{1} \subset \widetilde{H}$ that is, the subset $\widetilde{H}$ is invariant under $L_{\lambda}+$ $G(\cdot, \lambda)$. Moreover, the eigenvalues of $L_{\lambda}$ in $\widetilde{H}_{1}$ are $\lambda-4 n^{2}(1+$ $4 \gamma n^{2}$ ), which are simple. By the arguments in odd-periodic boundary condition we know that as $\lambda$ crosses the first critical value $4(1+4 \gamma), L_{\lambda}+G(\cdot, \lambda)$ bifurcates from $(0,4(1+4 \gamma))$ two steady states

$$
\begin{gathered}
u_{\lambda}(x)= \pm \alpha(\lambda) \sin 2 x+o(\alpha(\lambda)), \\
\alpha(\lambda)=\sqrt{\frac{4(\lambda-4(1+4 \gamma))}{3 \lambda}} .
\end{gathered}
$$

Because of the invariance of (79) for the translation

$$
u(x, t) \longrightarrow u(x+\theta, t), \quad \theta \in \mathbb{R},
$$

the functions

$$
u_{\lambda}(x+\theta)= \pm \alpha(\lambda) \sin 2(x+\theta)+o(\alpha(\lambda)), \quad \theta \in \mathbb{R}
$$

are steady states of (79). Moreover, the set

$$
\Gamma=\{\alpha(\lambda) \sin 2(x+\theta)+o(\alpha(\lambda)): \theta \in \mathbb{R}\}
$$

is a circle $S^{1}$ in $\widehat{H}$. Therefore,

$$
\begin{aligned}
\mathscr{A}_{\lambda}=\Gamma=\{ & a_{1}(\lambda) \sin 2 x+a_{2}(\lambda) \cos 2 x \\
& +o\left(\left|a_{1}(\lambda)\right|,\left|a_{2}(\lambda)\right|\right): a_{1}^{2}(\lambda) \\
& \left.+a_{2}^{2}(\lambda)=\frac{4(\lambda-4(1+4 \lambda))}{3 \lambda}>0\right\},
\end{aligned}
$$

where

$$
\alpha_{1}(\lambda)=\alpha(\lambda) \cos 2 \theta, \quad \alpha_{2}(\lambda)=\alpha(\lambda) \sin 2 \theta, \quad \theta \in \mathbb{R} .
$$

As $\lambda$ crosses the $n$th critical value $4 n^{2}\left(1+4 \gamma n^{2}\right)$, similar to the proof of Theorem 17, we can obtain the following.

Theorem 18. Equation (79) bifurcates from $\left(0,4 n^{2}\left(1+4 \gamma n^{2}\right)\right)$, on the right side of $\lambda=4 n^{2}\left(1+4 \gamma n^{2}\right)$, an invariant set $\Sigma_{\lambda} \subset \widehat{H}$. Furthermore, $\Sigma_{\lambda} \cong S^{1}$ consists of steady states of (79). 


\section{Acknowledgments}

This work was supported by the National Science Foundation for Young Scientists of China (Grant no. 11101304), and the Tian Yuan Special Foundation (Grant no. 11226137).

\section{References}

[1] G. T. Dee and W. van Saarloos, "Bistable systems with propagating fronts leading to pattern formation," Physical Review Letters, vol. 60, pp. 2641-2644, 1988.

[2] N. N. Akhmediev, A. V. Buryak, and M. Karlsson, "Radiationless optical solitons with oscillating tails," Optics Communications, vol. 110, no. 5-6, pp. 540-544, 1994.

[3] B. Buffoni, A. R. Champneys, and J. F. Toland, "Bifurcation and coalescence of a plethora of homoclinic orbits for a Hamiltonian system," Journal of Dynamics and Differential Equations, vol. 8, no. 2, pp. 221-279, 1996.

[4] P. Coullet, C. Elphick, and D. Repaux, "Nature of spatial chaos," Physical Review Letters, vol. 58, no. 5, pp. 431-434, 1987.

[5] J. Swift and P. C. Hohenberg, "Hydrodynamic fluctuations at the convective instability," Physical Review A, vol. 15, pp. 319-328, 1977.

[6] R. A. Fisher, "The advance of advantageous genes," Annals of Eugenics, vol. 7, pp. 355-369, 1937.

[7] A. Kolmogorov, I. Petrovsky, and N. Piskunov, "Etude de l'équation de la diffusion avec croissance de la quantité de matière etson application à un problème biologique," Bulletin Université d'Etat à Moscou, Série Internationale A, vol. 1, pp. 125, 1937.

[8] W. Zimmermann, "Propagating fronts near a Lifshitz point," Physical Review Letters, vol. 66, no. 11, p. 1546, 1991.

[9] J. B. van den Berg, "Uniqueness of solutions for the extended Fisher-Kolmogorov equation," Comptes Rendus de l'Académie des Sciences, vol. 326, no. 4, pp. 447-452, 1998.

[10] J. V. Chaparova, L. A. Peletier, and S. A. Tersian, "Existence and nonexistence of nontrivial solutions of semilinear fourthand sixth-order differential equations," Advances in Differential Equations, vol. 8, no. 10, pp. 1237-1258, 2003.

[11] P. Danumjaya and A. K. Pani, "Numerical methods for the extended Fisher-Kolmogorov (EFK) equation," International Journal of Numerical Analysis and Modeling, vol. 3, no. 2, pp. 186-210, 2006.

[12] M. A. Peletier, "Non-existence and uniqueness results for fourth-order Hamiltonian systems," Nonlinearity, vol. 12, no. 6, pp. 1555-1570, 1999.

[13] L. A. Peletier and W. C. Troy, "Spatial patterns described by the extended Fisher-Kolmogorov equation: periodic solutions," SIAM Journal on Mathematical Analysis, vol. 28, no. 6, pp. 13171353, 1997.

[14] J. Kwapisz, "Uniqueness of the stationary wave for the extended Fisher-Kolmogorov equation," Journal of Differential Equations, vol. 165, no. 1, pp. 235-253, 2000.

[15] L. A. Peletier and W. C. Troy, "Spatial patterns described by the extended Fisher-Kolmogorov (EFK) equation: kinks," Differential and Integral Equations, vol. 8, no. 6, pp. 1279-1304, 1995.

[16] L. A. Peletier and W. C. Troy, "A topological shooting method and the existence of kinks of the extended Fisher-Kolmogorov equation," Topological Methods in Nonlinear Analysis, vol. 6, no. 2, pp. 331-355, 1995.
[17] M. V. Bartuccelli, "On the asymptotic positivity of solutions for the extended Fisher-Kolmogorov equation with nonlinear diffusion," Mathematical Methods in the Applied Sciences, vol. 25, no. 8, pp. 701-708, 2002.

[18] D. Hilhorst, L. A. Peletier, and R. Schätzle, " $\Gamma$-limit for the extended Fisher-Kolmogorov equation," Proceedings of the Royal Society of Edinburgh A, vol. 132, no. 1, pp. 141-162, 2002.

[19] J. K. Hale, Asymptotic Behavior of Dissipative Systems, vol. 25 of Mathematical Surveys and Monographs, American Mathematical Society, Providence, RI, USA, 1988.

[20] L. A. Peletier and W. C. Troy, Spatial Patterns: Higher Order Models in Physics and Mechanics, vol. 45 of Progress in Nonlinear Differential Equations and Their Applications, Birkhäuser, Boston, Mass, USA, 2001.

[21] L. A. Peletier, W. C. Troy, and R. C. A. M. van der Vorst, "Stationary solutions of a fourth-order nonlinear diffusion equation," Differentsial'nye Uravneniya, vol. 31, pp. 327-337, 1995 (Russian).

[22] W. D. Kalies, J. Kwapisz, and R. C. A. M. van der Vorst, "Homotopy classes for stable connections between Hamiltonian saddle-focus equilibria," Communications in Mathematical Physics, vol. 193, no. 2, pp. 337-371, 1998.

[23] J. B. van den Berg, Dynamics and equilibria of fourth order dfferential equations [Ph.D. thesis], Leiden University, Leiden, Netherlands, 2000.

[24] W. D. Kalies, J. Kwapisz, J. B. van den Berg, and R. C. A. M. van der Vorst, "Homotopy classes for stable periodic and chaotic patterns in fourth-order Hamiltonian systems," Communications in Mathematical Physics, vol. 214, no. 3, pp. 573-592, 2000.

[25] W. D. Kalies and R. C. A. M. van der Vorst, "Multitransition homoclinic and heteroclinic solutions of the extended FisherKolmogorov equation," Journal of Differential Equations, vol. 131, no. 2, pp. 209-228, 1996.

[26] L. A. Peletier and W. C. Troy, "Chaotic spatial patterns described by the extended Fisher-Kolmogorov equation," Journal of Differential Equations, vol. 129, no. 2, pp. 458-508, 1996.

[27] J. B. van den Berg and R. C. A. M. van der Vorst, "Stable patterns for fourth-order parabolic equations," Duke Mathematical Journal, vol. 115, no. 3, pp. 513-558, 2002.

[28] T. Ma and S. Wang, Bifurcation Theory and Applications, vol. 53, World Scientific, Hackensack, NJ, USA, 2005.

[29] T. Ma and S. Wang, "Dynamic bifurcation of nonlinear evolution equations," Chinese Annals of Mathematics B, vol. 26, no. 2, pp. 185-206, 2005.

[30] T. Ma and S. Wang, "Dynamic bifurcation and stability in the Rayleigh-Bénard convection," Communications in Mathematical Sciences, vol. 2, no. 2, pp. 159-183, 2004.

[31] T. Ma and S. Wang, "Attractor bifurcation theory and its applications to Rayleigh-Bénard convection," Communications on Pure and Applied Analysis, vol. 2, no. 4, pp. 591-599, 2003.

[32] M. Yari, "Attractor bifurcation and final patterns of the $n$ dimensional and generalized Swift-Hohenberg equations," Discrete and Continuous Dynamical Systems B, vol. 7, no. 2, pp. 441456, 2007.

[33] Q. Huang and J. Tang, "Bifurcation of a limit cycle in the AC-driven complex Ginzburg-Landau equation," Discrete and Continuous Dynamical Systems B, vol. 14, no. 1, pp. 129-141, 2010.

[34] T. Ma, J. Park, and S. Wang, "Dynamic bifurcation of the Ginzburg-Landau equation," SIAM Journal on Applied Dynamical Systems, vol. 3, no. 4, pp. 620-635, 2004. 
[35] Y. Zhang, L. Song, and W. Axia, "Dynamical bifurcation for the Kuramoto-Sivashinsky equation," Nonlinear Analysis: Theory, Methods \& Applications, vol. 74, no. 4, pp. 1155-1163, 2011.

[36] A. Pazy, Semigroups of Linear Operators and Applications to Partial Differential Equations, vol. 44 of Applied Mathematical Sciences, Springer, New York, NY, USA, 1983.

[37] D. Henry, Geometric Theory of Semilinear Parabolic Equations, vol. 840 of Lecture Notes in Mathematics, Springer, Berlin, Germany, 1981. 


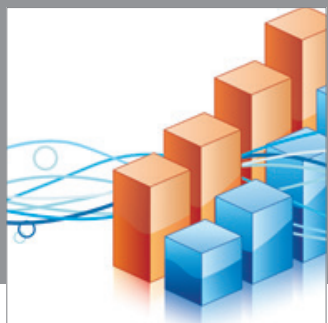

Advances in

Operations Research

mansans

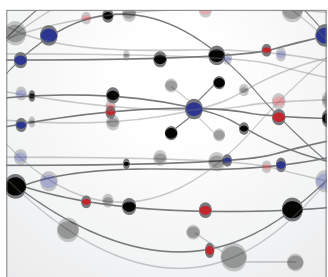

The Scientific World Journal
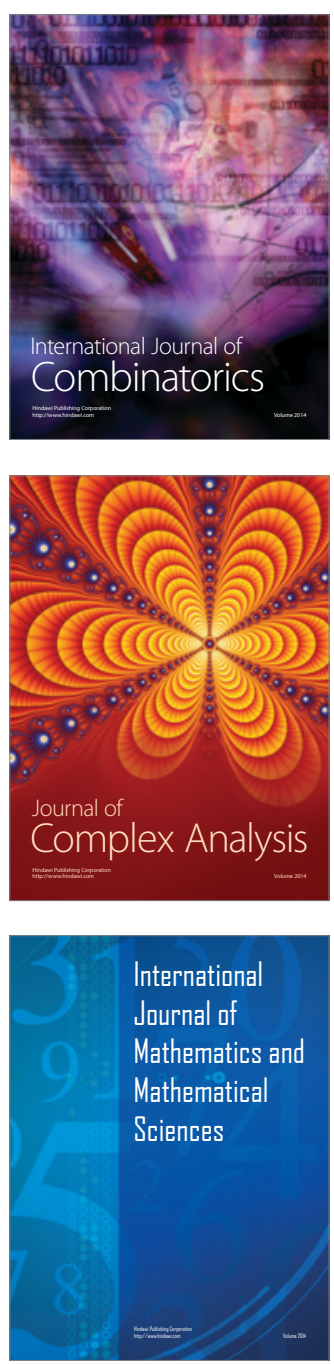
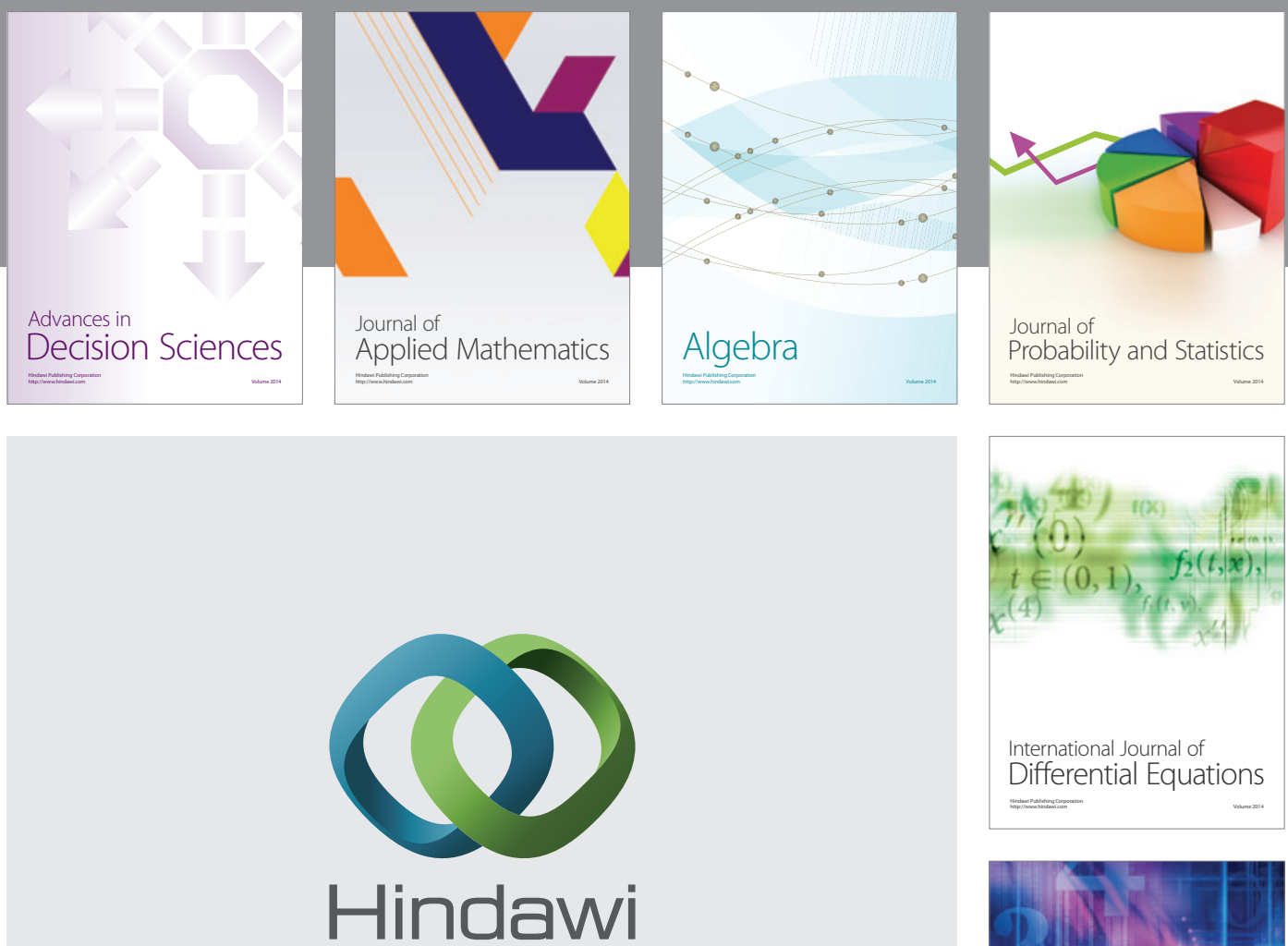

Submit your manuscripts at http://www.hindawi.com
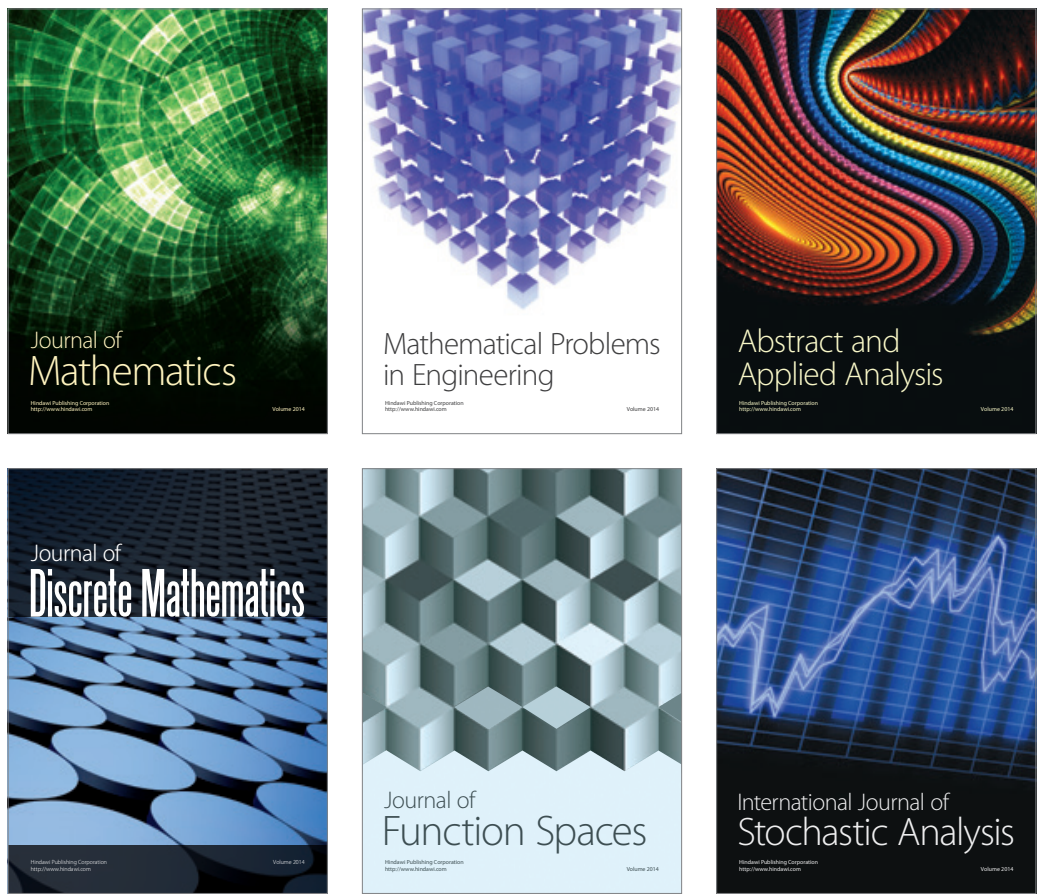

Journal of

Function Spaces

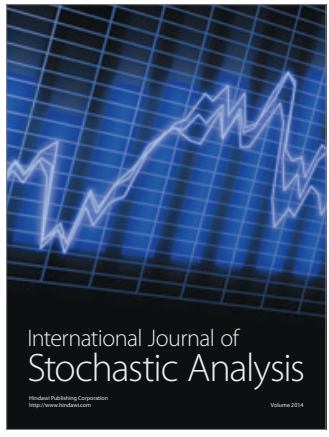

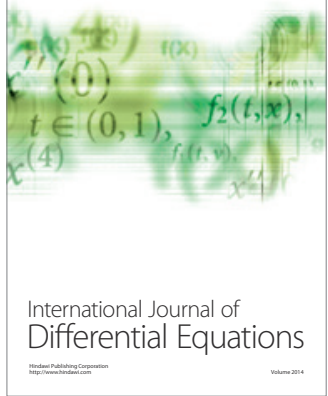
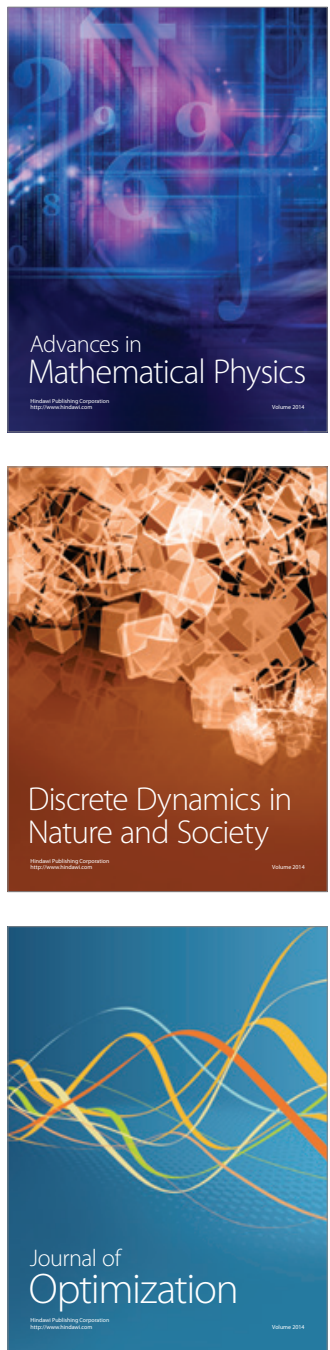